Projets

de paysage

\section{Projets de paysage}

Revue scientifique sur la conception et l'aménagement de l'espace

$8 \mid 2012$

Les concepteurs de jardins et de parcs japonais

\title{
Usages récréatifs et agriculture en espace périurbain au nord de Nantes
}

Tensions autour de la mise en place d'un périmètre de protection d'espaces agricoles et naturels

Recreational Uses and Agriculture in a Peri-urban Space in the North of Nantes - Tensions around the Setting up of a Marginal Protection Area of Agricultural and Natural Spaces

Cécile Rialland-Juin

\section{OpenEdition}

\section{Journals}

Édition électronique

URL : http://journals.openedition.org/paysage/15298

DOI : $10.4000 /$ paysage. 15298

ISSN : 1969-6124

\section{Éditeur :}

École nationale supérieure du paysage de Versailles-Marseille, Institut national des sciences appliquées Centre Val de Loire - École de la nature et du paysage, École nationale supérieure d'architecture et de paysage de Bordeaux, École nationale supérieure d'architecture et de paysage de Lille, Agrocampus Angers

Référence électronique

Cécile Rialland-Juin, "Usages récréatifs et agriculture en espace périurbain au nord de Nantes », Projets de paysage [En ligne], 8 | 2012, mis en ligne le 13 juillet 2012, consulté le 11 mars 2021. URL http://journals.openedition.org/paysage/15298; DOI : https://doi.org/10.4000/paysage.15298

Ce document a été généré automatiquement le 11 mars 2021.

Projets de paysage 


\section{Usages récréatifs et agriculture en espace périurbain au nord de Nantes}

Tensions autour de la mise en place d'un périmètre de protection d'espaces agricoles et naturels

Recreational Uses and Agriculture in a Peri-urban Space in the North of Nantes - Tensions around the Setting up of a Marginal Protection Area of Agricultural and Natural Spaces

Cécile Rialland-Juin

1 Depuis dix années, 550000 hectares de terres agricoles ont disparu en France, dont les deux tiers ont été destinés à l'urbanisation (enquête Teruti, Lucas, 2009). Dans un contexte d'étalement urbain, les territoires au nord de Nantes n'échappent pas à la règle et connaissent des dynamiques paysagères propres aux espaces périurbains.

2 Autrefois largement vouées à l'agriculture, les campagnes nantaises deviennent à la fois des espaces résidentiels, de desserrement d'entreprises mais aussi de loisirs et d'évasion (Renard, 2012). Les communes de la communauté de communes d'Erdre et Gesvres (CCEG) illustrent bien cette tendance. Dans un paysage agreste dominant, la présence d'un habitat dispersé hérité favorise les germes d'urbanisation. Un projet d'aéroport amplifie la pression sur les terres agricoles et engendre des concurrences entre les usages productifs et récréatifs. La mise en place d'un périmètre de protection des terres agricoles ${ }^{1}$ provoque des tensions entre les propriétaires de parcelles à chevaux et le milieu agricole. À partir d'une approche paysagère, on s'attachera à mesurer la part des activités récréatives au sein des espaces agraires périurbains. Cet état des lieux permettra ensuite d'interroger les acteurs et les enjeux autour de la question du foncier agricole. La finalité étant d'appréhender les conditions favorables à une gestion durable et différenciée des territoires. 


\section{Le territoire Nantes-Nord : un espace rural sous pression urbaine et confronté à un projet d'aéroport}

3 Le territoire analysé (figure 1), soit une douzaine de communes au nord de Nantes, est caractérisé par des surfaces agricoles importantes et une structure foncière héritée duale. De nombreuses métairies aux terres groupées sur les plateaux s'opposent aux petites propriétés des villages situés dans les vallées (Renard, 1976 et Palierne, 1980). Longtemps le secteur a été préservé de l'étalement urbain du fait de la présence de grands domaines et du projet aéroportuaire qui date de la fin des années 1960. Les agriculteurs encore en place pratiquent une agriculture conventionnelle de polyculture élevage reposant sur la production laitière intensive.

Depuis peu, ce secteur est confronté à une arrivée massive de population, avec des taux annuels de croissance de 2 à $3 \%$ (figure 2). Ici comme ailleurs ces populations entendent s'installer dans un espace rural afin de bénéficier d'aménités. Si nombre d'entre elles peuplent les lotissements accolés aux bourgs, elles sont aussi nombreuses à s'établir dans les villages et hameaux, recyclant l'habitat agricole d'autrefois (figure 3) ou faisant construire à proximité. Il s'ensuit un mitage généralisé lié aux structures agraires héritées, les municipalités ayant naguère mis en place des plans d'occupation des sols laxistes favorisant ce mitage de l'habitat.

L'acquisition ou la construction de résidences s'accompagnent de pratiques récréatives sur les terrains proches, souvent de petites parcelles embocagées, peu propices à une agriculture productive, mais aptes à accueillir des équidés, un étang ou un jardin d'agrément.

Figure 1. Le territoire Nantes-Nord : un espace sous pression urbaine et confronté à un projet d'aéroport

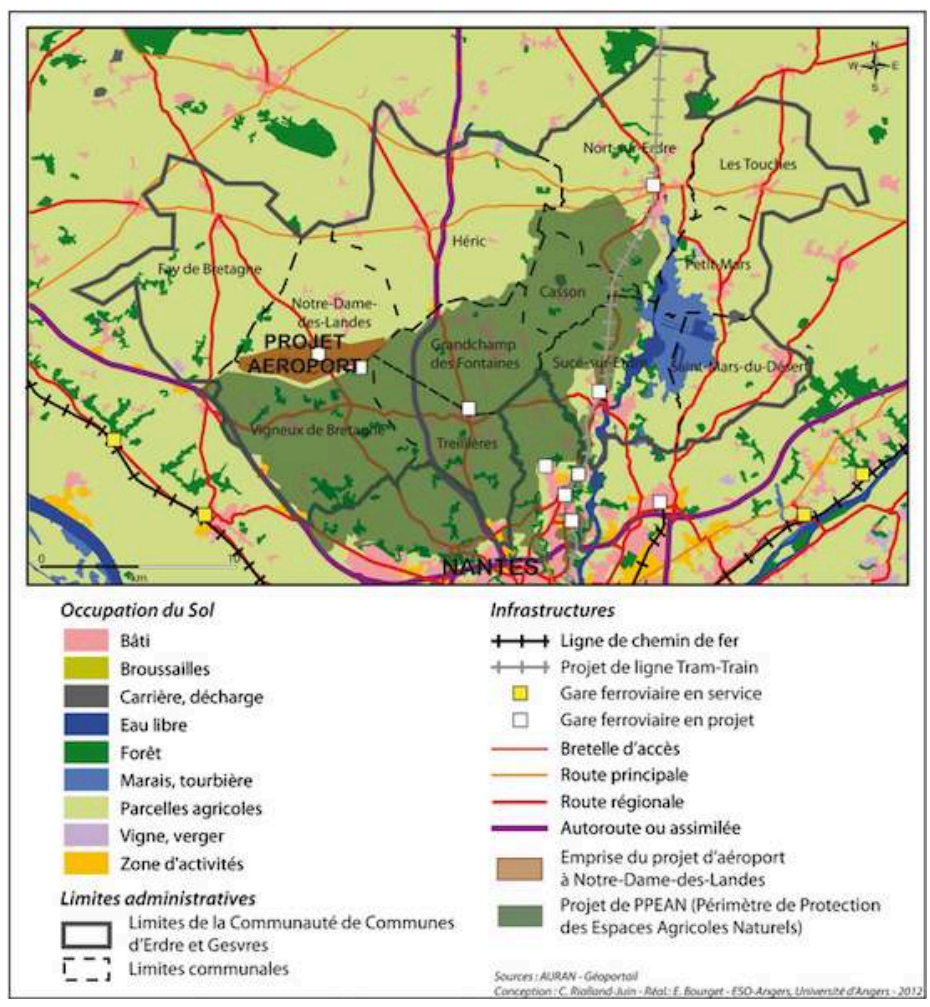


Figure 2. Évolution de la population 1999-2006 en Loire-Atlantique

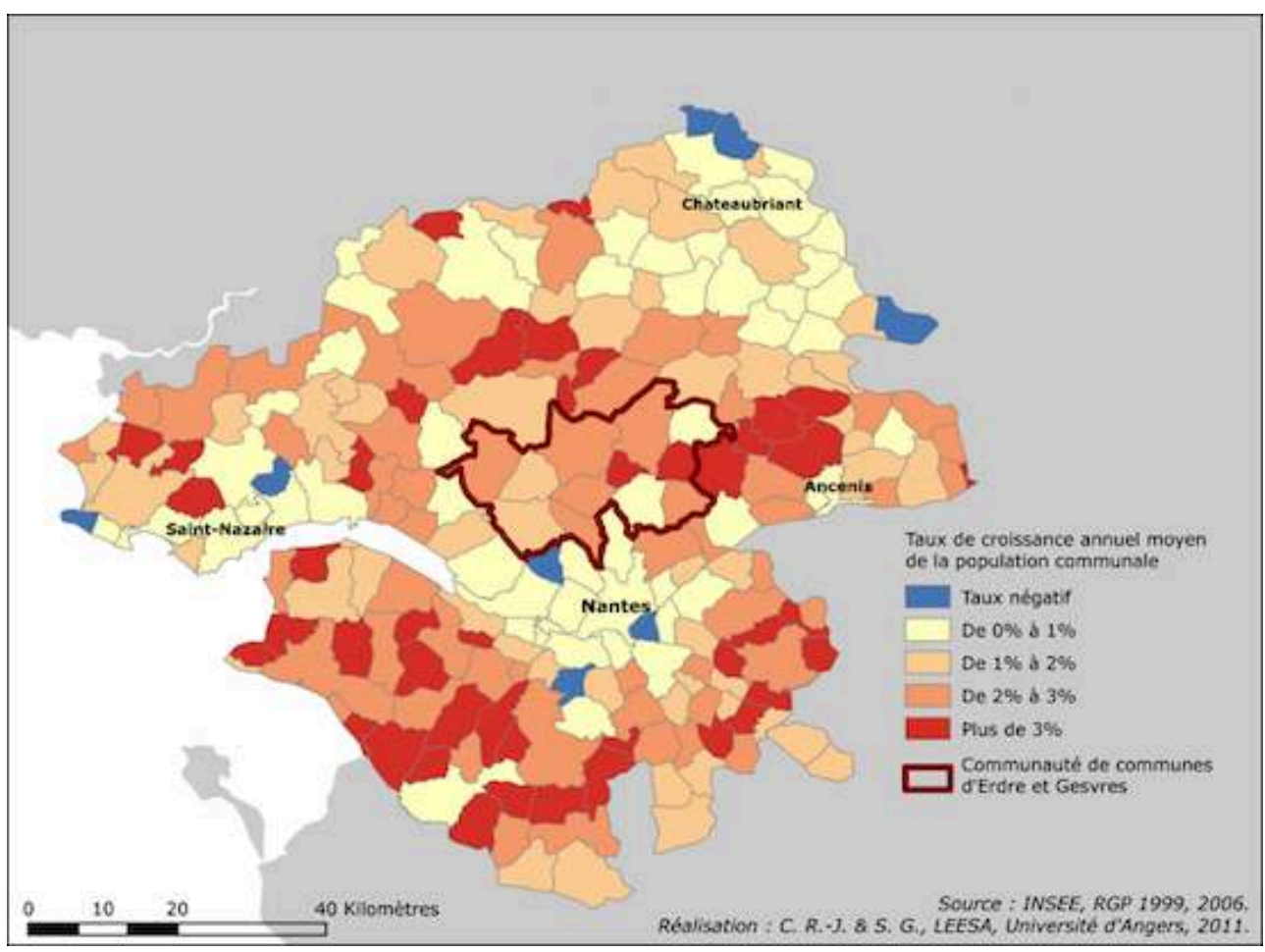

Figure 3. Une « longère », maison ancienne de hameau restaurée (Le Champ Rivaud, commune d'Héric)

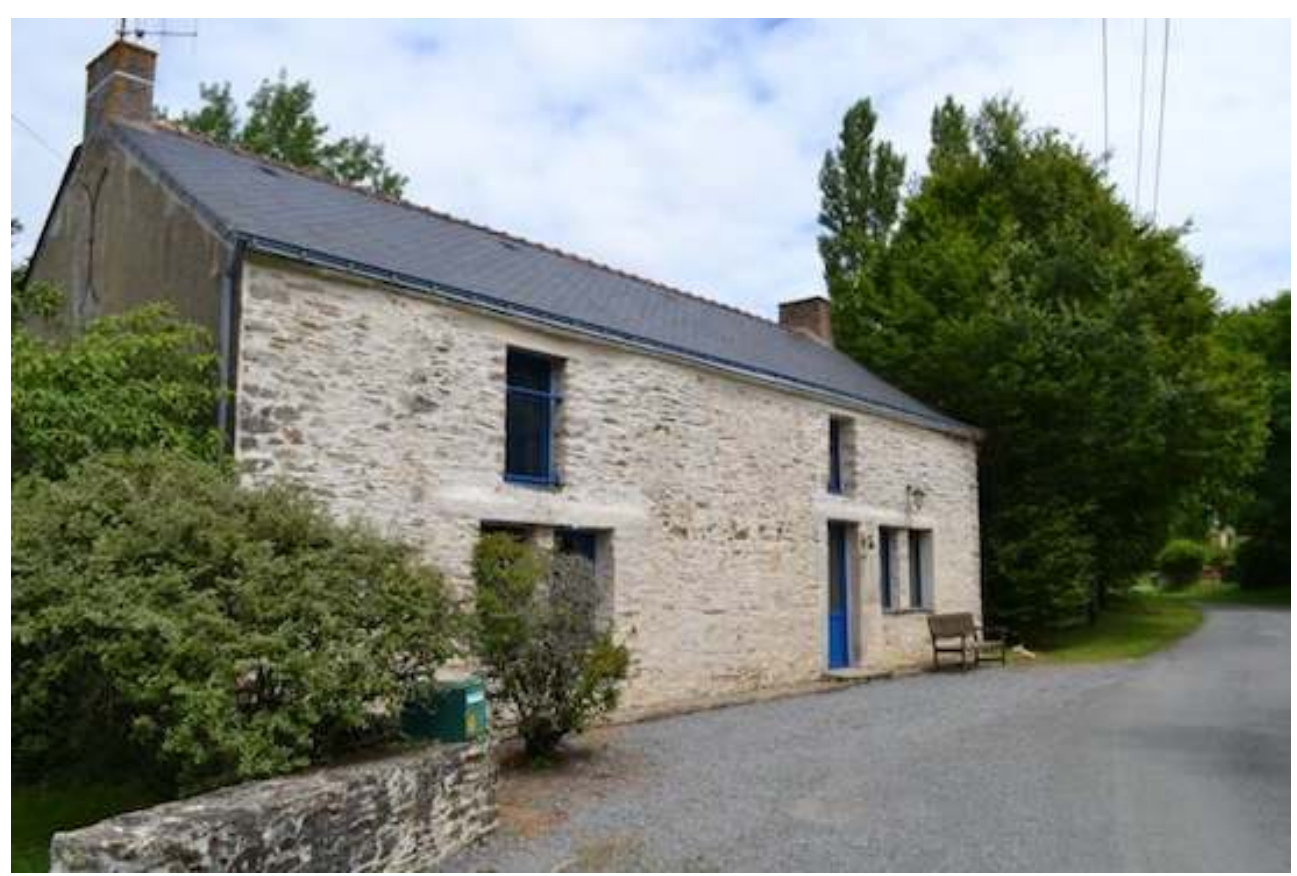

Source : Cécile Rialland-Juin, 2011. 


\section{Des usages récréatifs occultés face à la protection des espaces agricoles}

6 Le projet d'aéroport et la multiplication des usages récréatifs par les nouvelles populations sont à l'origine du projet de mise en place d'un périmètre de protection des espaces agricoles. Ce dernier, porté par la profession agricole, les services de l'État et les collectivités locales, est en cours de montage. Il se heurte aux nouveaux usages de loisirs dans la mesure où les terres utilisées par ces activités, notamment les parcelles à chevaux, ne sont pas reconnues comme terres agricoles de plein exercice. La menace d'expropriation pèse sur ces parcelles. Les autorités envisagent leur regroupement, voire leur suppression au sein des espaces qui deviendront uniquement réservés à l'agriculture.

7 Un observatoire du foncier est mis en place, animé par l'agence d'urbanisme de l'agglomération (AURAN), et le projet de PPEAN s'y inscrit. En revanche, les parcelles non productives incluses dans ces espaces agraires ne sont pas prises en compte. Les prairies accueillant des équidés (chevaux, poneys, ânes), les terrains d'agrément (avec un étang, un abri de jardin) se retrouvent englobés dans les surfaces agricoles. Un inventaire de ces usages non agricoles est donc à faire.

8 Au sein de la communauté de communes d'Erdre et de Gesvres, une association d'agriculteurs sans appartenance syndicale s'est mobilisée en 2010 afin de réaliser une cartographie des parcellaires des exploitations agricoles. L'objectif premier était de repérer les terres en friche afin de les récupérer pour un usage productif. Cette démarche a été contestée par les propriétaires de chevaux de loisirs qui ont organisé des manifestations, notamment dans la commune de Treillières où se trouvent de nombreuses parcelles à chevaux. L'ébauche de cartographie des parcellaires par les agriculteurs a le soutien de la chambre d'agriculture. Elle permet d'avancer que 500 hectares de friches sur 40000 hectares de SAU (superficie agricole utile) seraient récupérables ainsi que 300 hectares de parcelles destinées aux chevaux de loisirs. Audelà des chiffres avancés, qui restent à valider, c'est le principe d'associer les « friches récupérables » aux prairies accueillant des équidés qui heurte les adeptes des loisirs équestres.

9 L'inventaire des parcelles à équidés est du ressort des haras nationaux. Une réglementation datant du 25 juillet 2010 recense, pour des raisons sanitaires, les lieux d'accueil des équidés. L'accès à cette base de données est, pour des raisons de confidentialité, réservé aux services vétérinaires. Seuls les effectifs de chevaux à l'échelle d'un département sont diffusés, ce qui réduit son intérêt pour les études localisées.

10 À défaut de sources pertinentes, nous avons procédé à des relevés systématiques de terrain pour identifier les parcelles à usage récréatif. La démarche s'est accompagnée d'entretiens avec les responsables d'associations de loisirs liées aux chevaux, notamment les directeurs des centres équestres, poneys clubs et éleveurs de chevaux. Sur le territoire de la communauté de communes d'Erdre et Gesvres (CCEG) il existe une quinzaine de structures autour de la filière équine. L'élevage du cheval est une tradition locale, avec cinq hippodromes sur les treize que compte le département. Une vingtaine de centres équestres est installée dans la couronne périurbaine nantaise (Jousseaume, 2008). Ainsi, nous avons multiplié, ordonné et confronté les sources; 
méthode monographique indispensable pour donner un sens à des réflexions d'ordre théorique.

11 Faute de pouvoir parcourir l'ensemble du territoire de la communauté de communes, nous avons privilégié l'inventaire des catégories d'usage non agricole pour la commune d'Héric, vaste commune de 7000 hectares possédant plus de 4500 hectares en surface agricole. Les relevés effectués pendant l'été 2011 ont permis de classer en ordre décroissant les situations suivantes (figure 4):

- deux cents parcelles qui accueillent temporairement ou en permanence des équidés (chevaux, poneys, ânes) ;

- une vingtaine de parcelles de loisirs comportant un étang ;

- une dizaine de parcelles avec petits animaux (moutons, chèvres);

- une dizaine de jardins de loisirs isolés avec un cabanon ou caravane ;

- enfin deux parcelles occupées par des gens du voyage.

12 Les représentants de l'association locale de loisirs équestre estiment qu'il y aurait une centaine de chevaux sur la commune, et qu'il faut environ un hectare et demi par équidé. En surface, tous usages non directement agricoles confondus, il y aurait 200 hectares à soustraire de l'activité agricole. Sur un total de 4500 ha de terres agricoles, cette surface est relativement peu importante et amène à relativiser l'impact de ces activités récréatives.

13 En outre, la prise en compte de la localisation des parcelles apporte un bémol aux dommages que subirait l'agriculture du fait de leur présence. En effet, l'essentiel de ces parcelles se trouve à proximité immédiate des villages. Elles sont de petite taille et systématiquement entourées de haies, "là où il y a un cheval, il y a une haie » pour reprendre l'expression d'un responsable d'une association de loisirs équestres. Enfin, les villages encadrant généralement des vallées, les parcelles à chevaux correspondent à des prairies humides de fond de vallon. Ce type de parcelles ne convient pas à l'agriculture motorisée d'aujourd'hui. Rares sont donc les parcelles à chevaux localisées au sein même des parcellaires des exploitations agricoles professionnelles et susceptibles de peser sur les restructurations du foncier. À l'échelle de la commune d'Héric, nous n'avons relevé que cinq parcelles à être dans ce cas.

Ces constats, qui relativisent les désagréments subis par la profession agricole, n'empêchent pas les tensions latentes. Les propriétaires de chevaux de loisirs sont accusés de soustraire des terres qui pourraient être intégrées dans les exploitations agricoles. Sur une autre commune périurbaine de Nantes, à Saint-Étienne-de-Montluc, les professionnels agricoles ont par exemple mis en cause le mitage des surfaces agricoles par ces parcelles jugées, selon eux, improductives pour expliquer le refus du remembrement (Vernicolle, 1996). 
Figure 4. Présence de chevaux sur la commune d'Héric (Loire-Atlantique) en 2011

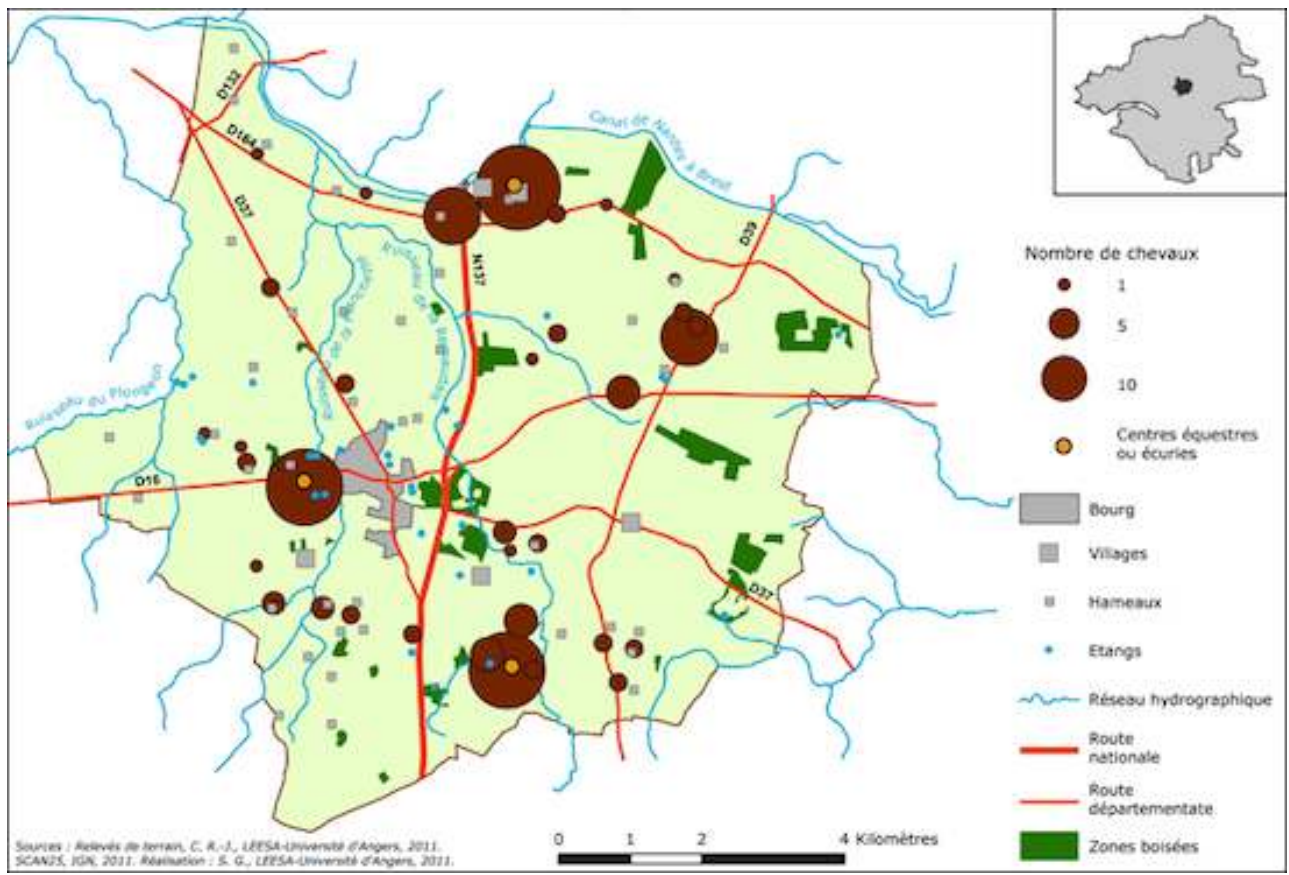

Outre la question de la distribution des parcelles dans l'espace agricole, se pose celle des pratiques de gestion. Les différentes situations ont été observées et classées en fonction de leur importance en trois catégories :

- Le propriétaire d'équidés est également propriétaire des pâtures. C'est la majorité des cas. Il s'agit alors de parcelles jointives à la résidence du propriétaire et souvent acquises avec celle-ci ;

- Le propriétaire de chevaux loue des parcelles, souvent par bail tacite et arrangement avec un agriculteur voisin, l'entretien de la parcelle et le partage de la récolte de foin étant le type d'accord le plus fréquent ;

- Plus rarement, il y a mise en pension des chevaux chez un exploitant agricole ou un centre équestre proche. Le propriétaire peut alors être citadin et pas seulement un périurbain récemment installé. C'est la solution la plus coûteuse. Le propriétaire de chevaux l'adopte quand il ne possède pas de foncier ou manque de temps pour s'occuper de ses animaux.

La première situation est la plus recherchée (Vial, 2011). Au sein des associations consultées, plus des deux tiers des propriétaires de chevaux possèdent leur propre pâture. Ce constat explique les tensions sur le marché du foncier, même si le prix des terres agricoles demeure faible en Loire-Atlantique ${ }^{2}$.

\section{Conflits d'usage entre les propriétaires d'équidés et le milieu agricole}

17 À la tension entre les différentes utilisations des espaces agraires, vient s'ajouter la réactivation du projet d'aéroport de Notre-Dame-des-Landes qui perturbe le marché foncier de tout le secteur analysé. Depuis les années 1960, le conseil général de la LoireAtlantique, porteur du projet, a procédé à l'acquisition de 850 hectares de terres agricoles. Ces dernières sont en grande partie relouées par baux précaires aux exploitants en place. Les inquiétudes sur la destination des terres proches de ce futur 
grand équipement sont réelles. Afin d'éviter une urbanisation désordonnée et de briser la spéculation, les pouvoirs publics, la profession agricole et la communauté de communes d'Erdre et de Gesvres ont initié la mise en place d'un périmètre de terres dont la vocation devrait être, pour plusieurs décennies, uniquement agricole et/ou naturelle. Ce projet ambitieux est en cours d'étude.

Dès la fin 2006, une réflexion a été engagée avec l'appui technique de l'agence d'urbanisme de Nantes (AURAN) pour établir les principes de protection à long terme. Les services de la chambre d'agriculture, alors aux mains de la Confédération paysanne, ont poussé le projet. L'idée d'un PPEAN, nouvel outil de gestion du foncier expérimenté en France, a peu à peu fait son chemin. En juillet 2009, un périmètre de 19300 hectares a été délimité. Il est situé entre l'emprise du projet d'aéroport et le front d'urbanisation de l'agglomération nantaise (figure 1). Ce périmètre de protection, sorte d'espace tampon, occupe vingt fois la superficie de la zone aéroportuaire. C'est beaucoup vis-àvis d'autres projets de PPEAN en France (Balny, Beth, Verlhac, 2009).

Dans ce périmètre, l'usage agricole est garanti. Seul un décret en Conseil d'État peut le remettre en cause. Les communes (elles sont une dizaine impactée par le PPEAN) s'engagent à intervenir sur le marché foncier par achat amiable ou droit de préemption sans autre contrainte que garantir l'usage agricole. C'est là que les tensions s'exercent. Il y a en effet possibilité d'expropriation dès lors que l'usage de certaines parcelles n'est pas exclusivement agricole. La mesure peut concerner les friches, volontaires ou non, les parcelles avec caravane ou mobil-home, mais également les parcelles à équidés. L'ensemble de ces biens, souvent aux mains de périurbains non-agriculteurs, peut faire l'objet d'une expropriation. On comprend les inquiétudes et le désarroi de ces acteurs qui s'estiment aussi légitimes que les agriculteurs pour préserver des espaces « naturels » aux portes de la ville.

Outre ce périmètre de protection des terres agricoles, un programme d'actions vise à favoriser une agriculture locale durable et diversifiée. Des outils comme les mesures agro-environnementales territorialisées, les contrats de conversion à l'agriculture biologique, la mise en place de filières courtes de type Association pour le maintien d'une agriculture paysanne (Amap) devraient être mobilisés. La volonté exprimée tant par les services publics que par la profession agricole est de disposer de surfaces d'un seul tenant, sans aucun mitage par d'autres usages. C'est d'autant plus difficile que l'on ne part pas de rien. Il s'agit d'un espace périurbain, peu ou pas remembré, où les nombreux hameaux ou villages sont autant de points d'ancrage à l'urbanisation.

21 Ce projet de sanctuarisation des espaces agricoles en milieu périurbain n'est pas sans rappeler d'autres exemples en France. On retrouve des modalités voisines de protection d'espaces agricoles dans la plaine du Var et dans la vallée de la Seine à l'aval de Paris. On peut souligner le cas emblématique du plateau de Saclay, au sud de Paris, cerné par l'urbanisation (dossier du Colos, 2006). L'agriculture demeure présente sur environ 2300 hectares, sous deux formes: des exploitations de grande culture et des exploitations en lien avec des marchés de proximité (loisirs, cueillette à la ferme, maraîchage). Un programme de maintien d'agriculture durable a été envisagé dès 1988 (charte du plateau). Il a abouti en 2012 à la délimitation d'une zone de protection naturelle agricole et forestière (ZPNAF) de 2624 hectares. Nous sommes loin des 19300 hectares du projet du PPEAN nantais.

À Nantes, la mise en œuvre du périmètre de protection des terres agricoles a suscité des oppositions et des conflits, notamment de la part des propriétaires de parcelles à 
chevaux. Mobilisés autour d'associations locales de loisirs équestres et de la fédération nationale Equiliberté ${ }^{3}$, ces usagers ont à plusieurs reprises exprimé leur mécontentement face aux menaces d'expropriation. Les arguments développés dans la presse et les sites Internet sont les suivants :

« Nous, propriétaires ou locataires de parcelles à chevaux, entendons conserver nos prairies et continuer à s'occuper nous-mêmes de nos chevaux. Autant par choix de vie que par souci économique, les coûts d'une pension étant élevés. Nous entretenons nos parcelles en respectant l'environnement. Il s'agit de pâturages extensifs et à vocation agricole. La filière équine représente un poids économique et social non négligeable pour nos territoires (fourrages, matériel, achat et vente de chevaux, aliments). Le Comité départemental du tourisme équestre en LoireAtlantique dénombre 2200 licenciés (soit près de la moitié des 4750 agriculteurs professionnels recensés en 2010). Enfin notre pratique du cheval assure le maintien d'une tradition équestre ancienne et fortement ancrée autour des hippodromes et des courses hippiques (Nort-sur-Erdre, Blain, Cordemais, Châteaubriant, Erbray, Plessé). » (Extrait d'un tract d'une association de loisirs équestres, février 2011.)

Interrogés individuellement sur leurs rapports avec les agriculteurs, la majorité d'entre eux reconnaît entretenir de bonnes relations. Les parcelles à chevaux, pour les différentes raisons évoquées plus haut (de petite taille, embocagées, proches des habitations, souvent très humides), ont longtemps été délaissées pour des impératifs techniques et économiques par les agriculteurs. L'introduction d'équidés sur ce parcellaire agricole a permis aux exploitants et aux propriétaires fonciers d'assurer l'entretien de leurs parcelles à moindre coût. Leurs différends avec les agriculteurs sont avant tout nés des discours officiels, tenus à une autre échelle, par les syndicats agricoles qui les désignent en partie responsables du déclin agricole du secteur.

Une conjonction de raisons alimente les inquiétudes et les conflits. Il faut rappeler que dans la région nantaise il y a très peu d'espaces naturels non "agricolisés " dans lesquels les usages récréatifs trouveraient aisément leur place. La faim de terres n'a pas disparu. La tendance à l'agrandissement des exploitations agricoles et l'emprise aéroportuaire ne font qu'accroître les tensions sur le marché foncier. Les besoins pour les réinstallations sont importants et il y a un effet domino sur les espaces proches. La profession agricole est ici depuis longtemps mobilisée sur la question foncière. Les exploitants entendent demeurer maîtres de la destination des terres rendues disponibles, surtout quand il y a menace sur l'organisation des parcellaires. Toute parcelle utilisée pour d'autres usages est alors considérée comme un bien qui leur échappe.

Dans ce conflit entre usages, il faut tenir compte des antagonismes provoqués par le clivage social. D'après les associations équestres consultées, la plupart des propriétaires de chevaux de loisir ne sont pas agriculteurs professionnels malgré leur adhésion à la mutualité sociale agricole et donc leur statut « d'exploitants agricoles ». Par ailleurs, la majorité (environ $60 \%$ ) appartient à des catégories sociales aisées. Ce constat laisse supposer des affinités politiques différentes de celles des pouvoirs locaux qui défendent la cause des milieux agricoles.

26 Enfin si l'idée de protéger les terres agricoles et les espaces naturels fait l'unanimité dans cet espace périurbain, les modalités pour y parvenir sont beaucoup moins consensuelles. La mise en œuvre de l'outil PPEAN suscite des critiques. Est-il bien adapté à des milieux dans lesquels les agriculteurs sont devenus très minoritaires face aux autres usagers? (Perrier-Cornet, 2002). Une concertation s'impose. Elle sera longue, à l'image de ce qui s'est passé sur d'autres sites comme le plateau de Saclay, car 
il s'agit de prendre en compte les intérêts de tous les acteurs, et ceux-ci peuvent être contradictoires.

\section{Conclusion}

Cette étude permet d'illustrer deux problèmes. Les espaces ruraux périurbains sont occupés par des catégories de population qui ont des représentations et des pratiques différentes de leur environnement (Le Caro, 2002). Les agriculteurs en place dans l'espace observé pratiquent pour la majorité d'entre eux une agriculture conventionnelle de polyculture élevage reposant sur une production laitière intensive. Ils sont confrontés à des demandes et à des usages qui ne sont pas dans leur logique. Ils ont besoin de surfaces d'un seul tenant sans autres activités perturbatrices. On comprend dès lors les inquiétudes des populations non agricoles qui aspirent à la pratique d'autres activités en milieu rural. Elles craignent, à juste titre semble-t-il, que le PPEAN réduise leurs possibilités d'usage de ces espaces. D'autant que le projet aéroportuaire vient parasiter les rapports, les autorités ayant à cœur de réduire les oppositions des milieux agricoles en favorisant la pérennité des exploitations.

Ensuite, l'analyse conduite ici démontre la nécessité de disposer, avant toute intervention, d'un état des lieux ordonné des réalités foncières et de la société locale. Rien ne peut se faire sans cette connaissance des enjeux et des acteurs. Face à un système de polyculture élevage intensif avec habitat dispersé, les problèmes nés de l'intrusion de nouveaux usages ne sont pas ceux que l'on observe dans des systèmes spécialisés ou de grande culture avec habitat groupé (Vial, 2009). D'un lieu à l'autre cependant, la gestion durable des paysages périurbains ne pourra faire l'économie d'un outil de type observatoire des dynamiques localisées (ODL, Renard, 1991) afin d'intégrer les multiples facteurs mais aussi d'impliquer tous les acteurs dans une gestion partagée et différenciée du territoire.

Seule la reconnaissance des usages multiples peut aboutir à des espaces ruraux durables. Les exclusions de type "sanctuaires agrestes ou naturels" s'avèrent inadaptées (Le Caro et al., 2007). Les espaces agraires proches des villes ont désormais des fonctions sociales diversifiées. Cet exemple montre qu'un partage raisonné et raisonnable, entre « gens d'ici et gens d'ailleurs », est la solution (Beuret, 1999).

\section{BIBLIOGRAPHIE}

Balny, P., Beth, O., Verlhac, E., Protéger les espaces agricoles et naturels face à l'étalement urbain, Paris, La Documentation française, 2009, $58 \mathrm{p}$.

Beuret, J.-E., « Petits arrangements entre acteurs... Les voies d'une gestion concertée de l'espace rural », Natures Sciences Sociétés, $n^{\circ}$ 1, vol. 7, Paris, 1999, p. 21-30.

Enquête Teruti-Lucas, « L'utilisation du territoire en 2009 », Agreste/ministère de l'Agriculture, $\mathrm{n}^{\circ} 213$, août 2010, $62 \mathrm{p}$. 
Jousseaume, J., « Une activité révélatrice des liens entre la ville et la campagne : les centres équestres de l'espace périurbain de l'agglomération nantaise », mémoire master 1 géographie, Igarun, Nantes 2008, 85 p.

Le Caro, Y., Madeline, P., Pierre, G., (sous la dir. de), Agriculteurs et territoires. Entre productivisme et exigences territoriales, Rennes, Presses universitaires de Rennes, coll. « Espace et Territoires », 2007, 268 p.

Le Caro, Y., Les loisirs en espace agricole. L'expérience d'un espace partagé, Rennes, Presses universitaires de Rennes, coll. « Géographie sociale », 2002, 431 p.

Palierne, J.-M., « Les héritages fonciers des paysages agraires du nord de la Loire-Atlantique », Cahiers nantais, $\mathrm{n}^{\circ} 3$, Igarun, Nantes, 1980, p. 13-28.

Perrier-Cornet, Ph. (sous la dir. de), À qui appartient l'espace rural ?, La Tour d'Aigues, Éditions de l'Aube, 2002, $143 \mathrm{p}$.

Renard, J., Les campagnes nantaises, un demi-siècle de révolutions sociales et paysagères 1960-2010, Rennes, Presses universitaires de Rennes, 2012, 192 p.

Renard, J., «L'avenir des terres agricoles dans l'Ouest, contribution à l'état des lieux », Norois, 1991, vol. 152, p. 393-396.

Renard, J., Les évolutions contemporaines de la vie rurale dans les campagnes nantaise, Sables d'Olonne, Éditions le Cercle d'Or, 1976, 432 p.,

Vernicolle, K., « Étude du parcellaire d'exploitation agricole de la commune de Saint-Étienne-deMontluc », mémoire MST - géographie Igarun Nantes, 1996, 96 p.

Vial, C., « Cheval et territoire : La population des amateurs, propriétaires d'équidés de loisir. Quatre études locales de terrain ", Annuaire ECUS 2009, les Haras nationaux, p. 49-52.

Vial, C., Aubert, M., Perrier-Cornet, Ph., « Les choix organisationnels des propriétaires de chevaux de loisir dans les espaces ruraux ", Économie rurale, n 321, janvier-février 2011, p. 549-573.

\section{Sites web consultés}

Institut français du cheval et de l'équitation : http://www.haras-nationaux.fr Association Equiliberté : http://www.equiliberte.org

Association Treillières à cheval : http://www.treillieres.fr/accueil-2273.html

Association Equi'Libre Chapelain : http://www.oms-chapelle-sur-erdre.org/index.php?

page $=$ club\&retour $=$ history $\&$ club $=7$

Association Loisirs équestres Ouest Erdre (ALEOE) : http://www.aleoe.org/

Association Agriculteurs d'Erdre et Gesvres : http://www.cceg.fr/

AURAN, Agence urbanisme région agglomération nantaise - Observatoire du foncier en LoireAtlantique : http://www.auran.org/

Collectif OIN Saclay : http://www.colos.info

Chambre agriculture Loire-Atlantique (charte pour la prise en compte de l'agriculture dans l'aménagement du territoire) : https://pays-de-la-loire.chambres-agriculture.fr/vos-chambres/ en-loire-atlantique/

Société d'aménagement foncier et d'établissement rural (Safer) : www.safer.fr/ 


\section{NOTES}

1. Le PPEAN (périmètre de protection des espaces agricoles et naturels) est défini par la loi du développement des territoires ruraux (DTR) du 23 février 2005, le PPEAN complète et renforce le principe de protection des zonages agricoles du document d'urbanisme (loi solidarité renouvellement urbain, 2001) ou encore de la zone agricole protégée (loi orientation agriculture, 1999) avec notamment l'ajout d'un programme d'actions en faveur d'une diversification agricole. Ce nouvel outil permet aux collectivités d'exercer un droit de préemption, voire d'expropriation pour garantir l'intégrité ou l'aménagement du foncier agricole. Une vingtaine de PPEAN sont en cours au plan national.

2. Selon la Safer (Société d'aménagement de foncier et d'établissement rural), en LoireAtlantique, le prix moyen en 2010 d'un hectare de terre agricole était de 1600 euros l'hectare contre une moyenne nationale de 5200 euros par hectare.

3. La fédération nationale Equiliberté est une association qui assure depuis 2011 la défense des intérêts des usagers de loisirs équestres.

\section{RÉSUMÉS}

Dans un contexte d'étalement urbain et de projet d'aéroport au nord de la métropole nantaise, la mise en œuvre par les pouvoirs publics d'un périmètre de protection des terres agricoles et naturelles a déclenché des conflits d'usage autour du foncier agraire opposant les tenants du milieu agricole poursuivant des objectifs de production à des propriétaires de parcelles à chevaux motivés par le loisir équestre. L'auteur s'interroge sur les sources et les méthodes d'analyse pour mesurer, à grande échelle, la part, les fonctions, les enjeux et les perspectives de cet usage récréatif dans l'espace agricole et périurbain au nord de Nantes.

In a context of urban sprawl and airport project north of the city of Nantes, the implementation by authorities of a perimeter of protection of agricultural land and natural sparked conflicts over land use land opposing advocates of pursuing agricultural production targets to horse owners of plots motivated by leisure riding. The author examines the sources and analytical methods to measure, scale, unit, functions, challenges and opportunities of this recreational use only in agricultural and suburban areas north of Nantes.

\section{INDEX}

Mots-clés : paysage périurbain, parcellaire récréatif, foncier agricole, conflits d'usage

Keywords : suburban landscape, parcel recreation, agricultural land, use conflicts

\section{AUTEUR}

\section{CÉCILE RIALLAND-JUIN}

Maître de conférences en géographie rurale à l'université d'Angers, elle est enseignante au sein du master « Paysage et médiation » d'Agrocampus Ouest et chercheur au sein du Leesa 
(Laboratoire d'études environnementales des systèmes anthropisés), un des cinq sites de l'UMR 6554 du LETG (Littoral environnement télédétection géomatique).

cecile.rialland-juin[at]univ-angers[dot]fr 